\title{
Adaptive System Based on DSP for Active Noise Control
}

\author{
Jiang Wei, a \\ ${ }^{1}$ Shenyang Ligong University, Shenyang, Liaoning, 110159, China \\ awilljiang2008@sohu.com
}

Keywords: Adaptive System, Active Noise Control, Noise Reduction.

\begin{abstract}
Active noise control is combined with adaptive controller to construct adaptive active noise control. Adaptive active noise control is primarily researched in the theory and structure. DSP is applied in adaptive controller to design adaptive system for active noise control. Adaptive system can steadily operate in noise environment and noise reduction is monitored to analyze.
\end{abstract}

\section{Introduction}

Active noise control is an advanced technology of noise control, and it is different in essence from passive noise control. Active noise control is based on interference cancellation of sound. Secondary noise source generates secondary sound whose amplitude is same and whose phase is opposite as primary sound generated by primary noise source. Secondary sound cancels primary sound so that noise is reduced [1].

Adaptive active noise control is on the basis of active noise control. It tracks the change of noise environment and monitors the effect of noise reduction. It continually trains the parameters of adaptive controller by adaptive algorithm and adjusts secondary noise source in real time. Primary noise source is effectively cancelled by secondary noise source so that optimal noise reduction is obtained. How to design adaptive controller and efficiently achieve adaptive algorithm is the key of adaptive active noise control. Digital signal processor(DSP) not only computes in high speed, but also it contains rich hardware source and powerful development tool [2]. In this paper DSP is applied in adaptive controller for achieving adaptive algorithm. Adaptive system based on DSP for active noise control is designed and constructed. When adaptive system is operating in noise environment, noise reduction is obtained to monitor.

\section{Adaptive Active Noise Control}

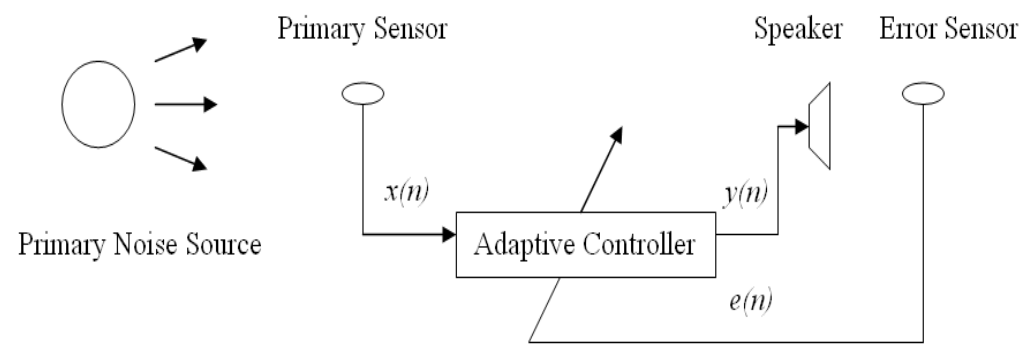

Fig.1 Schematic diagram of adaptive active noise control

Adaptive active noise control is shown in Fig.1. Primary sensor is located in primary noise source, which measures primary signal $x(n)$ at $\mathrm{n}$ time. Primary signal $x(n)$ inputs adaptive controller, then the controller parallel computes to output secondary signal $y(n)$. Secondary signal $y(n)$ drives speaker to make secondary noise source that cancels primary noise source [3]. Error sensor detects error signal $e(n)$, then $e(n)$ is fed back to the controller. Adaptive controller is modified by means of error signal $e(n)$, then secondary signal $y(n)$ is adjusted in order that error signal $e(n)$ will be the least. 


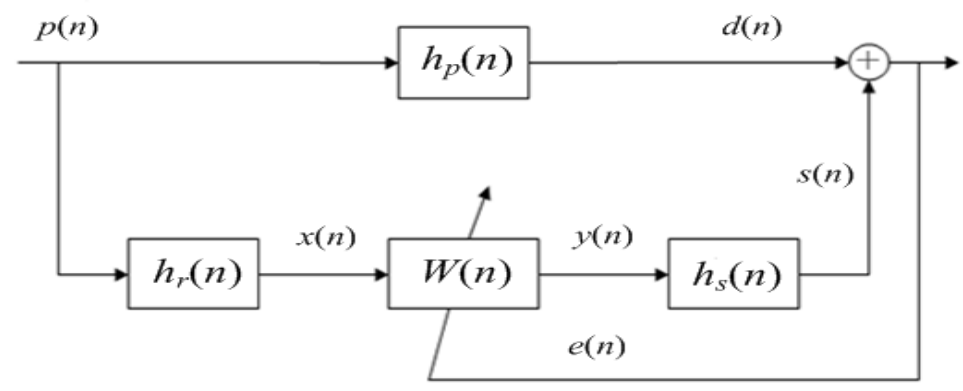

Fig.2 Block diagram of adaptive active noise control

Fig. 2 shows block diagram of adaptive active noise control. At $\mathrm{n}$ time $h_{r}(n)$ is the transfer function from primary noise source to primary sensor, $h_{p}(n)$ is the transfer function from primary noise source to error sensor, and $h_{s}(n)$ is the transfer function from speaker to error sensor [4]. $p(n)$ is primary noise, $x(n)$ is primary signal and $d(n)$ is expected signal. $x(n)$ and $d(n)$ are obtained as follows:

$x(n)=p(n) * h_{r}(n)$,

$d(n)=p(n) * h_{p}(n)$,

where $*$ is convolution. At $n$ time $W(n)$ is a weight vector of adaptive controller and $X(n)$ is an input vector of the controller, which are described as follows:

$$
\begin{aligned}
& W(n)=\left[\begin{array}{lll}
w_{1}(n), & w_{2}(n), \ldots, & w_{L}(n)
\end{array}\right]^{T}, \\
& X(n)=[x(n), \quad x(n-1), \ldots, \quad x(n-L+1)]^{T},
\end{aligned}
$$

where $L$ is the length of the controller. $y(n)$ is secondary signal and $s(n)$ is secondary noise, which are obtained as follows:

$$
\begin{aligned}
& y(n)=X^{T}(n) W(n)=\sum_{i=1}^{L} w_{i}(n) x(n-i+1), \\
& s(n)=y(n) * h_{s}(n)=\left(X^{T}(n) W(n)\right) * h_{s}(n)=\left(X(n) * h_{s}(n)\right)^{T} W(n) .
\end{aligned}
$$

$R(n)$ is defined as follows:

$$
\begin{aligned}
& R(n)=X(n) * h_{s}(n), \\
& R(n)=[r(n), \quad r(n-1), \quad \ldots, \quad r(n-L+1)]^{T} .
\end{aligned}
$$

Secondary noise $s(n)$ is redefined as follows:

$$
s(n)=R^{T}(n) W(n)=\sum_{i=1}^{L} w_{i}(n) r(n-i+1) .
$$

Error signal $e(n)$ is given as follows:

$$
e(n)=d(n)+s(n)=d(n)+R^{T}(n) W(n) .
$$

Adaptive controller uses least mean square (LMS) algorithm. Based on gradient descent theory, at $n$ time the correction of weight vector $W(n)$ is derived as follows:

$$
\nabla(n)=\frac{\partial E\left[e^{2}(n)\right]}{\partial W(n)}=2 e(n) R(n) .
$$

At $n+1$ time the adaptation rule of weight vector $W(n)$ is updated as follows:

$W(n+1)=W(n)-u \nabla(n)=W(n)-2 u e(n) R(n)$,

where $u$ is a factor of stability and convergence, $0<u<1$.

\section{Adaptive System based on DSP}

Adaptive system based on DSP for active noise control includes signal generation, signal acquisition, executive device and adaptive controller. Fig. 3 shows block diagram of adaptive system based on DSP. Signal generation consists of signal generator, power amplifier and speaker. Signal acquisition consists of primary sensor, error sensor and preamplifier. Executive device consists of power amplifier and speaker. The core of adaptive controller is main control computer and DSP, the other part of which includes antialiasing filter, $\mathrm{S} / \mathrm{H}, \mathrm{A} / \mathrm{D}, \mathrm{D} / \mathrm{A}$ and reconstruction filter. 


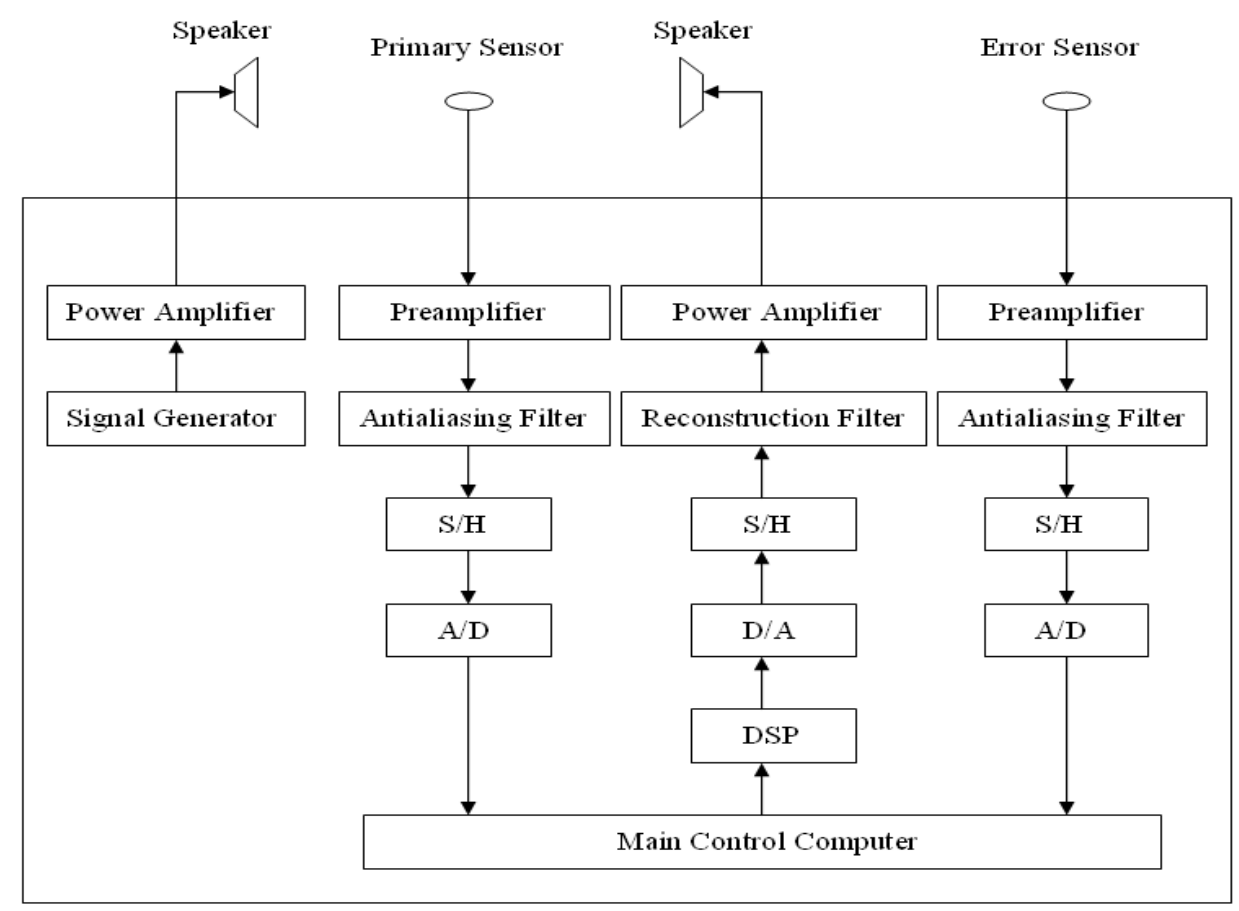

Fig.3 Block diagram of adaptive system based on DSP

First of all, signal generator drives speaker through power amplifier to make primary noise source. Primary sensor extracts primary signal through preamplifier and antialiasing filter, then primary signal converts into primary digital signal through $\mathrm{S} / \mathrm{H}$ and $\mathrm{A} / \mathrm{D}$. Primary digital signal inputs main control computer, then it is transferred to DSP. DSP outputs secondary signal through D/A, S/H and reconstruction filter, then secondary signal drives speaker through power amplifier to make secondary noise source. Error sensor extracts error signal through preamplifier and antialiasing filter, then error signal converts into error digital signal through $\mathrm{S} / \mathrm{H}$ and $\mathrm{A} / \mathrm{D}$. Error digital signal inputs DSP, and DSP adjusts secondary signal. Finally secondary signal drives speaker to modify secondary noise source. Main control computer and DSP jointly manage, and moreover, they parallel communicate to increase operational speed. Main control computer collects to store primary signal and error signal. DSP runs adaptive algorithm to update controller weight and adjusts secondary signal [5].

\section{Noise Test}

Fig. 4 shows the configuration of adaptive system for noise test. Two speakers are collocated side by side in the centre of laboratory, and their distance is $0.5 \mathrm{~m}$. Two speakers separately make primary noise source and secondary noise source. Primary sensor is collocated right ahead of primary noise source, and their distance is $0.05 \mathrm{~m}$. Error sensor is collocated on the perpendicular bisector of two noise sources, and the distance is $0.05 \mathrm{~m}$ from error sensor to the midpoint of two noise sources. A semicircle is made, whose centre point is the midpoint of two noise sources, whose radius is $1.8 \mathrm{~m}$ and height is $1 \mathrm{~m} .13$ test points are collocated on the semicircle, and the angle of every two test points is 15 degrees [6].

Adaptive controller doesn't run, primary noise source only works and secondary noise source doesn't work. 13 test points separately measure sound pressure level of noise signal. Adaptive controller normally runs, and two noise sources both work. When adaptive system for active noise control is steadily operating, 13 test points measure sound pressure level of noise signal again. Primary noise source use $200 \mathrm{~Hz}$ and $300 \mathrm{~Hz}$ single frequency sine signal. The curves of sound pressure level of $200 \mathrm{~Hz}$ and $300 \mathrm{~Hz}$ sine signal are shown in Fig.5. 


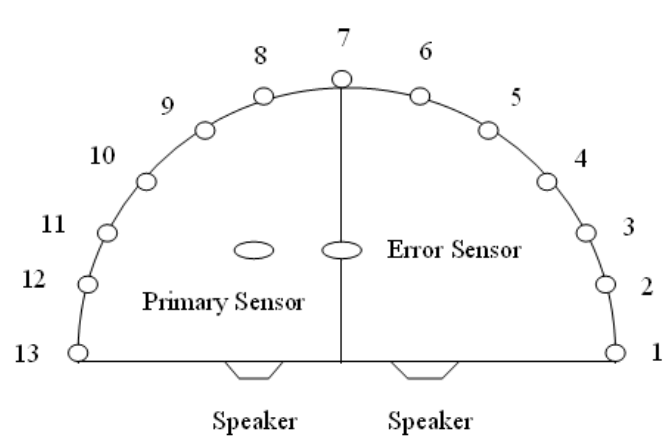

Fig.4 Configuration of adaptive system for noise test

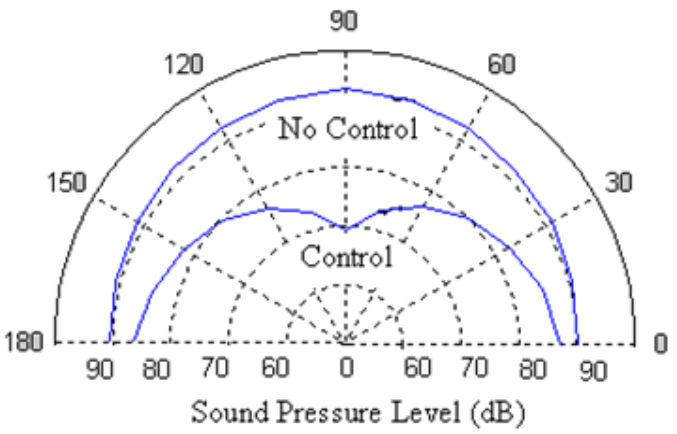

(a)

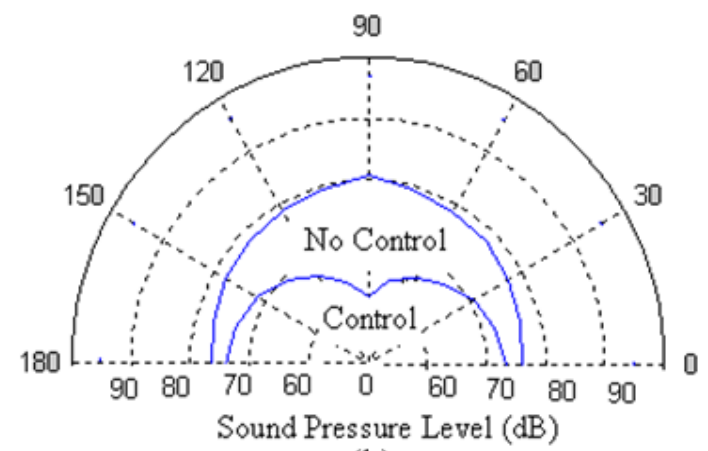

(b)

Fig.5 Curve of sound pressure level of sine signal (a) $200 \mathrm{~Hz}$ (b) $300 \mathrm{~Hz}$

\section{Conclusions}

The test results are completely summarized to analyze the trend of noise reduction. The effect and cause of noise reduction are researched based on the curves of sound pressure level of noise signal. Noise reduction is more obvious in the range of 90 degrees ahead of primary noise source and secondary noise source. Noise reduction is the most obvious on the perpendicular bisector of two noise sources, because the perpendicular bisector is where secondary sound can perfectly match with primary sound for the amplitude and phase. Noise reduction is $24.3 \mathrm{~dB}$ and $19.8 \mathrm{~dB}$ on the perpendicular bisector for $200 \mathrm{~Hz}$ and $300 \mathrm{~Hz}$ sine signal. Noise reduction is poorer in farther range from the perpendicular bisector of two noise sources. That is because the sound transmits to attenuate so that secondary sound can't match well with primary sound. Noise reduction is $3.4 \mathrm{~dB}$ and $2.5 \mathrm{~dB}$ in farthest range from the perpendicular bisector. Average noise reduction is $11.9 \mathrm{~dB}$ and $9.2 \mathrm{~dB}$ in the test range for $200 \mathrm{~Hz}$ and $300 \mathrm{~Hz}$ sine signal. The test analysis illustrate that adaptive system based on DSP for active noise control can effectively cancel primary noise source, and moreover, adaptive system can steadily operate to obtain desired noise reduction.

\section{References}

[1] Kean Chen: Adaptive Active Noise Control (National Defense Industry Press, China 2005).

[2] Xiongwei Zhang, Liang Chen: Development and Application of DSP (Publishing House of Electronics Industry, China 2002).

[3] Jiang Wei: Advanced Materials Research Vol.457-458 (2012), p. 196

[4] Youpeng Zhang, Chunming Chen, Guohai Li: Modern Electronics Technique 17 (2011), p. 104

[5] Qingjun Meng, Shenggang Yan: Measurement and Control Technology 9 (2006), p. 31

[6] Jiang Wei: Microcomputer Information 27 (2011), p. 76 